\title{
CURRENT SURVEYING METHODS FOR THE INTEGRATION OF ADDITIVE MANUFACTURING IN THE CONSTRUCTION PROCESS
}

\author{
Mehdi Maboudi ${ }^{1 *}$, Markus Gerke ${ }^{1}$, Norman $\mathrm{Hack}^{2}$, Leon Brohmann ${ }^{2}$, Patrick Schwerdtner ${ }^{3}$, Gerrit Placzek ${ }^{3}$ \\ ' Institute of Geodesy and Photogrammetry, Technische Universität Braunschweig, (m.maboudi, m.gerke)@tu-braunschweig.de \\ 2Institute of Structural Design, Technische Universität Braunschweig, (n.hack, leon.brohmann)@tu-braunschweig.de \\ Institute of Construction Engineering and Management, Technische Universität Braunschweig, (patrick.schwerdtner, \\ g.placzek)@tu-braunschweig.de
}

\section{TS : Digital twins}

KEY WORDS: Additive Manufacturing, 3D Concrete Printing, Surveying, Digital Twin, BIM, Geometric Inspection

\begin{abstract}
:
The Technical University of Braunschweig (Brunswick) and Technical University of Munich were successful to establish a Collaborative Research Centre called "Additive Manufacturing in Construction (AMC)- The Challenge of Large Scale" starting from 2020 and funded by the German Research Foundation (DFG). The aim of this project is "to create the basic conditions for the introduction of additive manufacturing in construction, and thus to pave the way for the use of resource-efficient constructions with a high level of design freedom". Surveying engineering (geodetic surveying, photogrammetry, laser scanning and GNSS) plays a major role in one of the sub-projects called "Integration of Additive Manufacturing in the Construction Process". This paper aims at introducing the large scale AMC with the main focus on investigating the role of surveying engineering in this topic which will be a topic of high interest in the coming years in the digital fabrication within construction field. After a short introduction on additive manufacturing in construction, this paper will present the general aims and structure of the Collaborative Research Centre. Thereupon, the importance of geometric quality inspection and establishing and transferring different coordinate systems during the Additive Manufacturing (AM) construction steps (elements fabrication, installation and whole structure/building control) and the role of geodetic surveying, photogrammetry, laser scanning and GNSS will be outlined. This will be presented within a subproject called "C06: Integration of Additive Manufacturing in the Construction Process" and potentials and challenges for integrating surveying engineering in component and building level additive manufacturing in construction are mentioned.
\end{abstract}

\section{INTRODUCTION}

The Technical University of Braunschweig (Brunswick) and Technical University of Munich were successful to establish a Collaborative Research Centre called "Additive Manufacturing in Construction (AMC)- The Challenge of Large Scale" starting from 2020 and funded by the German Research Foundation (DFG). The aim of this project is "to create the basic conditions for the introduction of additive manufacturing in construction, and thus to pave the way for the use of resource-efficient constructions with a high level of design freedom". This project consists of 20 sub-projects and 24 institutes are involved in this long term project (initially planned for 12 years in 3 phases). Surveying engineering (geodetic surveying, photogrammetry, laser scanning and GNSS) plays a major role in one of the subprojects called "Integration of Additive Manufacturing in the Construction Process". This paper aims at introducing the large scale AMC with the main focus on investigating the role of surveying engineering in this topic which would be a topic of high interest in the coming years in the digital fabrication within construction field.

While most other industries, particularly in the areas of materials and computational design, have experienced remarkable improvements over the last few decades and benefit from a very high level of digitization, automation and data exchange, building construction sector has been following similar conventional techniques for the structural works (e. g. pouring in-situ concrete into the formworks) which leads to a stagnant labor productivity. This unimpressive track record can be attributed to various internal and external challenges: Lack of innovation and delayed adoption, poor design and engineering, availability of low-priced manpower, risk of lowering quality and durability, to name a few (Kloft et al. 2019; "World Economic Forum").

\section{ADDITIVE MANUFACTURING IN CONSTRUCTION}

3D printing is an additive manufacturing technique of building up 3D products layer by layer. Using previously defined target geometries, the computerized calculation of the layer structure and the printing paths will be conducted. This technology has the potential to save resources because the structure can be reduced to the minimum amount of material that is necessary to fulfill the structural requirements. Furthermore, 3D printing provides a high degree of freedom in form and shape which offers fabrication of various components with customized characteristics resulting in unprecedented flexibility in overall building design in order to fulfill the exact expectation of the designers ("Arup" 2019; Bos et al. 2016; Hack 2018). "The innovative potential of AMC is essentially based on the combination of so far conflicting qualities that could not be merged in the past: Freedom of form and industrial production; Automation and Individualization; and Resource-efficiency and economics." (Kloft et al. 2019)

In this article, as the first phase of C06, we will focus on $3 \mathrm{D}$ concrete printing (3DCP). The $3 \mathrm{D}$ concrete printing approach 
could be generally categorized in the following three groups: particle bed-binding; material extrusion; and, material jetting. Each uses a digital model to drive the process where sequential layers of material are bonded together to form a physical object. Some samples of AMC products are depicted in Figure 1.

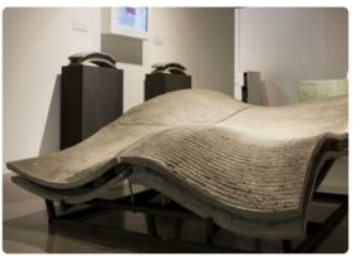

c

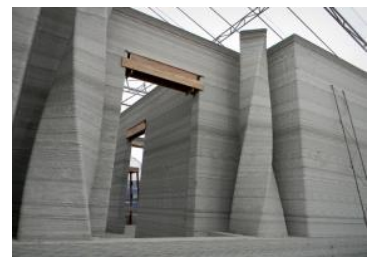

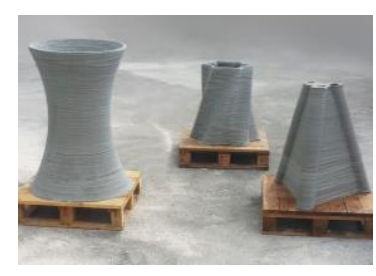

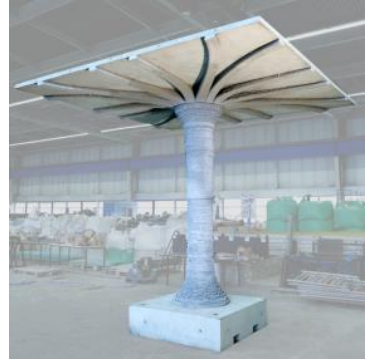

b

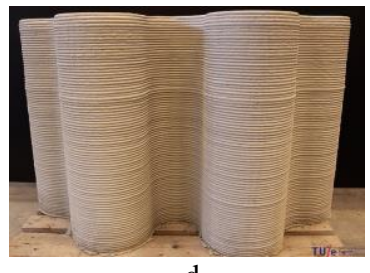

d

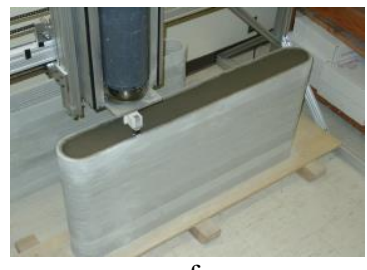

f
Figure 1. Some 3DCP samples: a) geometrical variations on posts and columns. Image: Courtesy of Xtree; b) Shotcrete $3 \mathrm{D}$ printed ribbed slab on a printed column, Image: Courtesy of ITE/TU Braunschweig; c) Horizontal component manufacturer. Image: Courtesy of Loughborough University, UK; d) Wavelike surface of additively manufactured concrete walls, Image Courtesy: Eindhoven University of Technology, e) 3D-printed building, Image: Courtesy of Totalkustom, USA; f) Full size wall built with contour crafting technology, Image: courtesy of B. Khoshnevis http://contourcrafting.com/

Before implementing AM in construction, some fundamental challenges should be considered. Most importantly, the successful transfer of well-established small Scale AM technologies to the large scale of construction projects, the variety of materials, the effect on resource efficiency and high degree of flexibility and freedom in the shape needed for the fabricated buildings' elements should be investigated. Hence, all production steps consisting design, manufacturing and quality control methods must be fundamentally rethought in order to realize the full potential of additive manufacturing in construction. Multilateral effects of object size, production speed, surface quality, and dimensional accuracy which are completely different from those of the 3D precision printing of smaller elements should be considered in this new setup.

\subsection{Structure of the "Additive Manufacturing in Construction" Collaborative Research Center}

The general structure of the research program of the collaborative research center "Additive Manufacturing in Construction (AMC)- The Challenge of Large Scale" which is reported mainly from the application of the project is shown in Figure 2 and is structured into three focus areas:

- Area A: Materials and Processes: Basic principles for the development of unique AMC processes for concrete, steel, and timber will be investigated in this area.

- Area B: Computational Modeling and Process Control: Development of models and discretization schemes for numerical simulation approaches on the one hand and advancing robotic fabrication on the other hand will be investigated.

- Area C: Design and Construction: The most important subjects related to the implementation of AM in the process chain of design and construction will be addressed in this focus area. The interaction between physical objects and their digital twins connects the focus areas A and C. Hereafter in this paper; we call the subprojects in Area $\mathrm{A}, \mathrm{B}$, or $\mathrm{C}$ as $\mathrm{A}, \mathrm{B}$, or $\mathrm{C}$ projects.

Focus area $\mathrm{C}$ provides feedback to the $\mathrm{A}$ projects by investigating novel design techniques and structural optimization as well as innovative assembling principles for additively manufactured structural elements. Moreover, the investigation of the seamless digitalization in construction and any information about building information modeling (BIM) will be discussed in this research area. Of particular interest for this paper are the effects associated with the implementation of innovative $\mathrm{AM}$ processes in the construction industry which will be addressed in one of the C-projects (C06). 


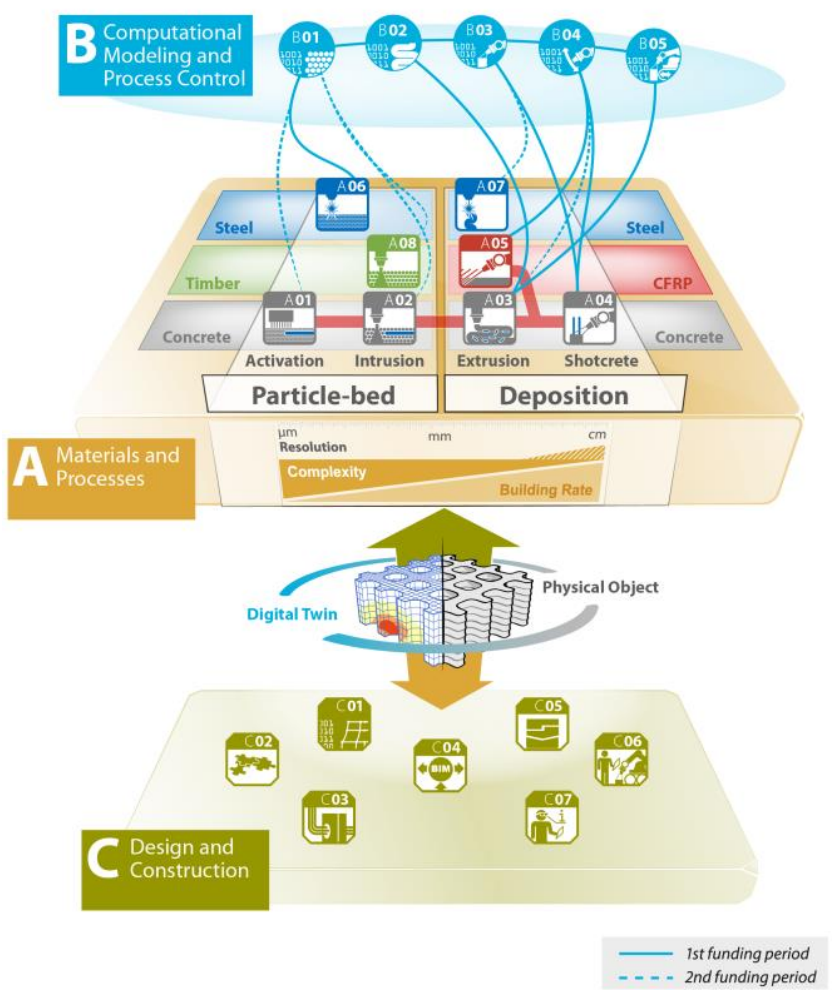

Figure 2. Structure of the Collaborative Research Center, Additive Manufacturing in Construction (Kloft et al. 2019)

\section{INTEGRATION OF ADDITIVE MANUFACTURING IN THE CONSTRUCTION PROCESS}

Bi-directional information flow provides the framework for the integration of additive manufacturing in construction in this project and will be investigated on three different scales: Firstly, on the scale of the building component, by investigating construction parameters, methods of sensorbased quality assessment and techniques for product improvement using advanced scanning technology and machine learning. Secondly, on the scale of the building, by looking at the processes in the entire production chain and on-site assembly strategies, including - besides others unmanned aerial vehicles (UAV) for as-built monitoring and mixed reality (MR) applications for the assembly of AM components. Thirdly, on the scale of the construction industry, with an investigation of the implications of reducing the separation between design, planning and execution in a traditional sequential project delivery system. Here, digital tools enable continuous and uninterrupted quality control throughout the entire construction process (see Figure 3).

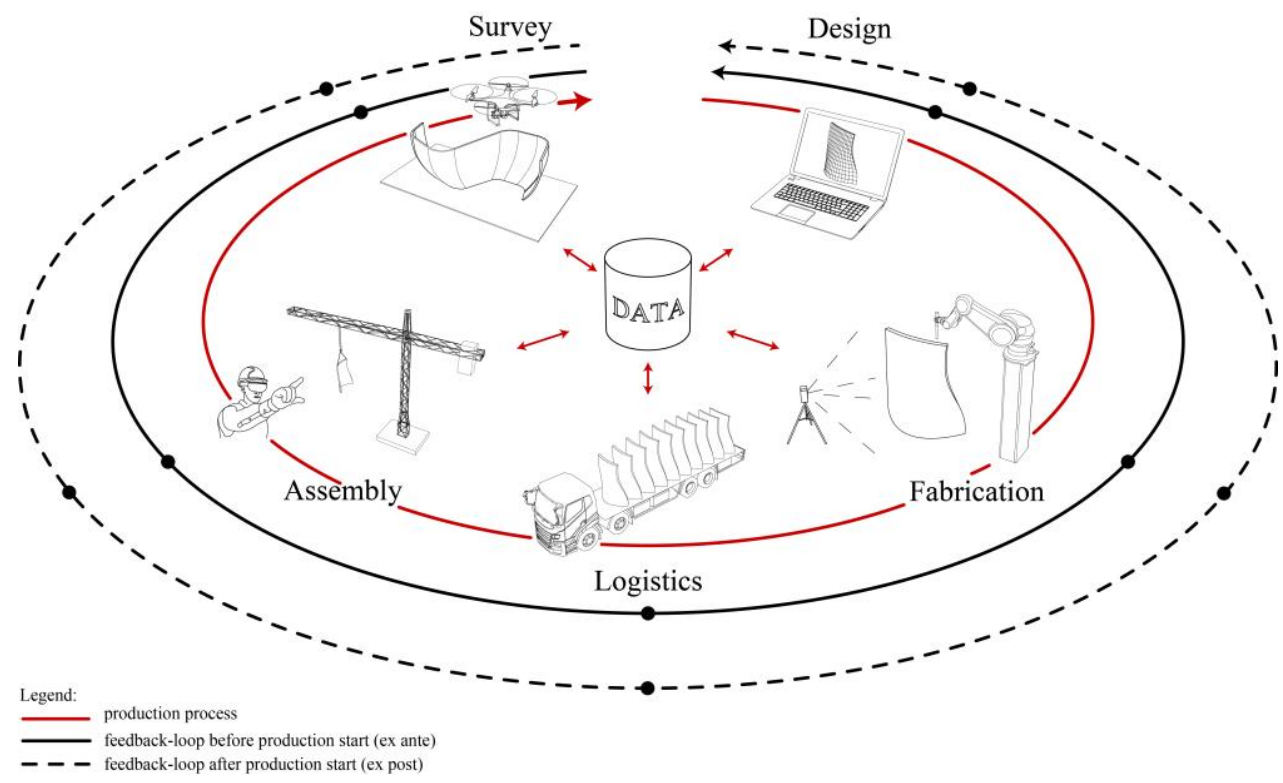

Figure 3 AM-based process chain for off-site fabrication 


\subsection{Component Level}

The manufactured component using all 3DCP approaches should be a facsimile of the 3D planned model (Buswell et al. 2018). Hence, dimensional conformity of the additively manufactured components to the as-planned model and specified tolerances and also the surface quality of the final product should be quantified. There are different approaches for reality capture in $3 \mathrm{DCP}$, which depend on component scales, component's shape complexity, construction stages, ambient environmental conditions and other technical or economic demands. Classic approaches like using a total station are applicable just for simple components. However, laser scanning and photogrammetry-based approach also including structured light-based methods are expressed as the most dominant data capturing approaches (Maboudi, et al. 2018; Kim et al. 2019). The main challenges and questions in component level quality assessment should be investigated. Object properties, environmental conditions, and data capturing technique as well as qualification of the data capturing and processing personnel may affect the results. Moreover, different aspects (geometry, surface quality) of the fabricated component should be observed. Additionally, the expected efficiency of the different setups (time, automation, cost) should be also considered. The most important challenges are:

- Material properties: Although in the current phase of the project, only concrete structures will be investigated, different conditions of the concrete can also affect the selection of the sensor and the achieved results. During maturing of the concrete, which the object is made of, the object would not be static necessarily and its reflectivity may change. Hence data capturing has to be carried out with special considerations.

- Different sizes and shapes of the printed object, and also accessibility/visibility of different parts of the objects should be considered for selecting the appropriate inspection method. Very tall objects or objects with overhangs are good examples in this context.

- Environmental condition: Usually the printing area is dusty and humid which may hinder the performance or even the possibility of data capturing. Moreover, due to security standards for some printing instruments, all sensor installation or capturing preparations during printing in the fabrication area is prohibited.

- Optimal QA technology (laser, structured light, multi-view photogrammetry): Considering various parameters related to the object, environment, sensor capabilities (both quality and speed), and requested specifications of the final output, different data capturing technologies should be investigated and the optimal one should be selected.

- Data acquisition mode (static, mobile or hybrid) should also be considered. Especially when some part of the component cannot be captured because of the nature of the common measurement techniques which are able to record the information just along the line of sight of the sensor.

- Existence of various surface defects should be checked. Hence, different surface quality assessment techniques should be investigated for reliable detection of defects in additively manufactured building components.
This defect detection may also be done during the surface finishing phase to improve the efficiency of this step.

- Co-registration of the measurement (As-Built data) with the 3D model of the object (As-planned) must be conducted before any further step. Feature based methods, or rough coregistration of two entities followed by a coarse registration like ICP (Besl and McKay 1992), or employing clearly defined identical points on printed component and 3D model, or utilizing well-defined points on the fabrication/printing environment (external targets) will be investigated.

\subsubsection{Preliminary study}

In order to investigate the capabilities of current approaches for measuring the deviation of printed building components from designed 3D models, and also to better understand the practical problems in this new environment, different objects with various specifications were printed using shotcrete $3 \mathrm{D}$ printing technology (SC3DP) at ITE's Digital Building Fabrication Laboratory (DBFL) at TU Braunschweig. Laser scanning and multi-view photogrammetry were used to generate two 3D point clouds of the printed component. Data was acquired under different object conditions (dry/wet, with/without reinforcement, before/after surface finishing) in order to take possible conditions on real construction projects into account. Figure 4 depicts a reinforced curved concrete component which was used for a preliminary analysis.
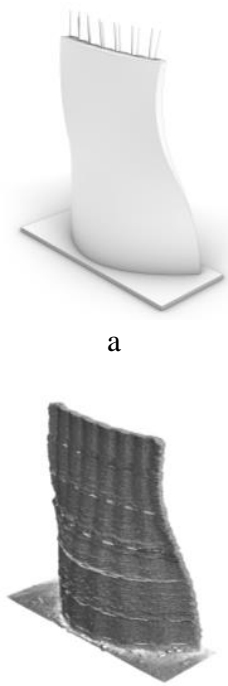

c

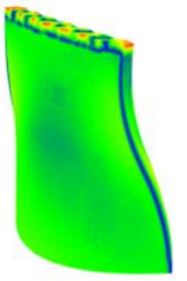

e

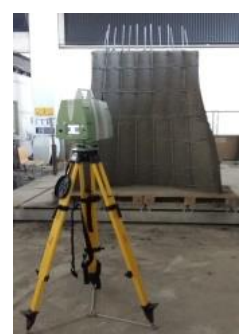

b

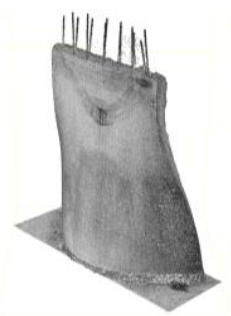

d

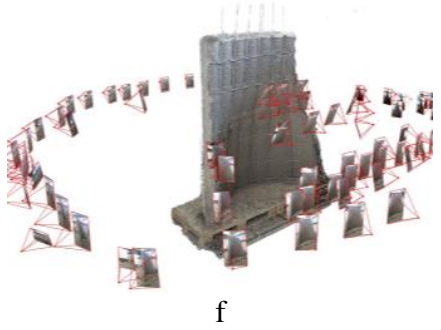

Figure 4. Shotcrete 3D printed demonstrator geometric inspection, (a) 3D designed model of the building component, b) TLS data acquisition, c) TLS Scan of the printed core, and d) of the finished component, e) printed component colorized by deviation of the printed component from designed 3D model, f) photogrammetric block around the fabricated component. 


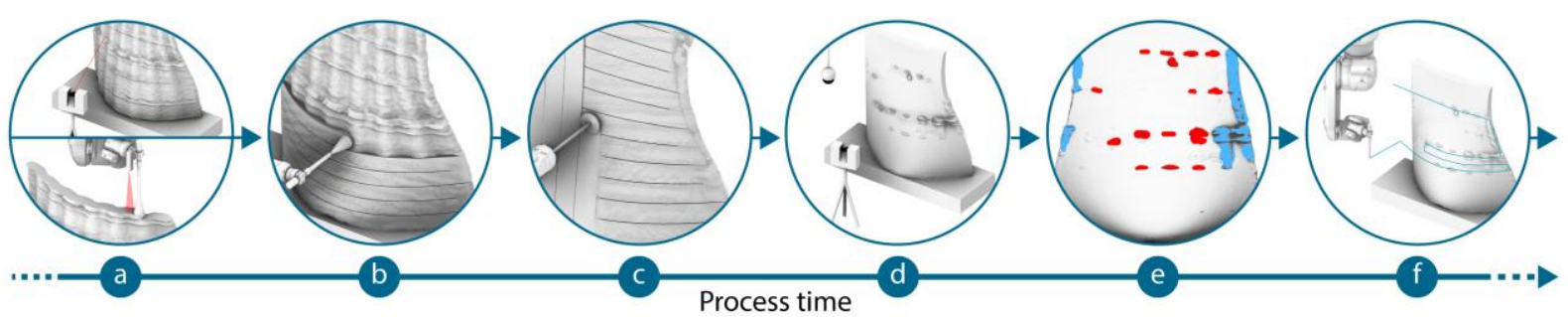

Figure 5. Process steps of a surface improvement process of a SC3DP- component: a) initial data acquisition of the given surface, b) second layer printing, c) post processing, d) data acquisition of the post processed surface; e) defect classification, and f) local post processing, based on the defect classification.

A multi-scale investigation and review on the geometric inspection methods for 3D Concrete Printing is co-authored and will be presented in the Digital Concrete 2020 conference (Buswell et al. 2020).

In comparison with common concrete extrusion techniques, where the profile of the deposited layers defines the resulting surface quality, the shotcrete $3 \mathrm{D}$ printing process allows to print secondary layers on the already printed object. During this process defects can be compensated, amplified or new ones could occur. They can appear by uneven distribution of printing material, wrong printing parameters or solely by the geometry and orientation of the printed surface. Additionally, obstructions can be caused by added elements like reinforcement or already underlying errors.

Not only could the defects be introduced during printing, but also during post-processing. Subtractive methods can cause concrete to break off during the process, especially if it matured too much. The detection of defects is therefore time sensitive. In contrast to the preliminary printed demonstrator, seen in Figure 4, in which the post-process parameters were laboriously set manually, Figure 5 depicts an early concept of an autonomous surface improvement process of a SC3DPcomponent. Due to the alternating additive and subtractive processing steps, multiple data acquisitions are to be considered and tested. Furthermore, the data-acquisition and inspection process should be also integrated into the automated post process.

\subsection{Building Level:}

In the building level, different aspects including global and updated local coordinate system, component identification, advanced methods for components assembly and effective data exchange with BIM must be considered and investigated. This feedback-loop is crucial in order to transfer in-situ information into the design and manufacturing processes, where the information can be used to make necessary adjustments before printing the next components (Schwerdtner 2018).

One additional key issue and challenge is the transformation of the "building model" coordinate system to the global measurement coordinate frame. One approach is using a GNSS-based system for establishing a reference system outside the construction site. Then the established coordinate system will be used to monitor the structure movement. Moreover, by installing proper reflectors on GNSS receivers and performing a resection using robotic total stations, the transformation parameters to the local (building) coordinate system in the current floor will be defined and updated regularly. By this means the GNSS-based localization is a proxy to realize the building coordinate system, even if salient points on site are not accessible. Within the building coordinate system, different data capturing devices and techniques like total stations, terrestrial laser scanners, structured light scanners or UAV-based surveys will be used to localize different components of the construction site, accurately. The established coordinate system would also serve other machines in the project, for example localizing mobile robots that will be used in another sub-project (B-05).

In addition to establishing the local coordinate system in the current level, identification of the specific component should be performed for its assembly. Installing RFID during or after printing could be a solution for this step. However, due to symmetry and self-similarity of many of the building components, and also to increase the automation of the whole process, identification and point cloud to model coregistration could be combined using at-least four well distributed markers on the surface of the object. Similar strategies are already utilized in the car and other industrial industries.

During assembly mixed reality (MR) enables the spatially correct visualization of virtual components within the surrounding environment (Hübner et al. 2018). Superimposing the digital model of building components on the physical environment provides a clear understanding of the relationship between the $3 \mathrm{D}$ designed model and the actual work on site. Trimble has recently announced a new wearable mixed reality hard hat, the Trimble XR10 with a Microsoft HoloLens (Figure 6), specifically designed for the construction industry ("Trimble Xr10" 2019). After installation of the component, final measurement and updating the model should be done. Classical as-isdocumentation techniques like laser scanning and UAV photogrammetry could be used to capture the data after assembly.

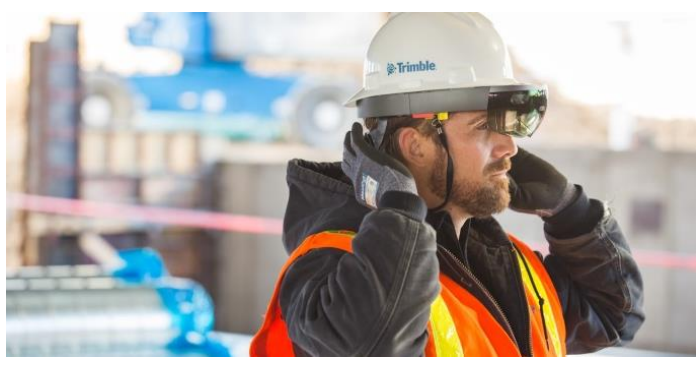

Figure 6. A prototype of a Trimble XR10 helmet with a Microsoft HoloLens https://constructible.trimble.com/ 
Although the appropriate extensions of the BIM standard regarding AM processes will have been investigated by another sub-project of the established CRC, time-stamped output of the surveying measurements should be exchanged with BIM, appropriately. So, proper interfaces and modeling strategies should be defined. Aspects to be considered will be amongst others: differential vs. full model transfer; modeling differences in a volumetric vs. a boundary representation. Furthermore, the relevant strategies and compiled information can be used to increase the functionality and efficiency of other subprojects. For example, some other subprojects which employ robots could benefit from the updated information from reality capturing methods.

\section{ACKNOWLEDGMENTS:}

This research is funded by the German Research Foundation (DFG) - TRR 277/1 2020 - Project number 414265976. The authors would like to thank the DFG for the support within the SFB/Transregio 277 - Additive manufacturing in construction. (Subproject C06)

\section{REFERENCES}

“Arup.” 2019. 2019. https://www.arup.com/.

Besl, P. J. , McKay, N. D. 1992. “A Method for Registration of 3-D Shapes." IEEE Transactions on Pattern Analysis and Machine Intelligence 14 (2): 239-56.

Bos, F. , Wolfs, R., Ahmed, Z., Salet T. 2016. "Additive Manufacturing of Concrete in Construction: Potentials and Challenges of 3D Concrete Printing." Virtual and Physical Prototyping 11 (3): 209-25.

Buswell, R. A., Kinnell P., Xu J., Hack N., Kloft H., Maboudi M., Gerke M., Massin P., Grasser G. , Wolfs R., Bos F. 2020. "Inspection Methods for 3D Concrete Printing." In Digital Concrete $2020-2^{\text {nd }}$ RILEM International Conference on Concrete and Digital Fabrication.
Buswell, R. A., Leal de Silva, W. R., Jones, S. Z., Dirrenberger J. 2018. "3D Printing Using Concrete Extrusion: A Roadmap for Research." Cement and Concrete Research. doi.org/10.1016/j.cemconres.2018.05.006.

Hack, N. 2018. "Mesh Mould: A Robotically Fabricated Structural Stay-in-Place Formwork System." ETH Zurich. doi.org/10.7134/phlebol.14-06.

Hübner, P., Weinmann, M., Wursthorn, S. 2018. "MarkerBased Localization of the Microsoft Hololens in Building Models", IAPRS - XLII-1: 195-202. doi.org/10.5194/isprsarchives-XLII-1-195-2018.

Kim, M. K., Wang, Q., Li, H. 2019. "Non-Contact Sensing Based Geometric Quality Assessment of Buildings and Civil Structures: A Review." Automation in Construction. doi.org/10.1016/j.autcon.2019.01.002.

Kloft, H, Gehlen C, Hack, N., Henke, K., Lowke, D., Mainka J. 2019. "Proposal for the Establishment and Funding of a Collaborative Research Centre (TRR 277), Additive Manufacturing in Construction (AMC) The Challenge of Large Scale.”, https://www.tu-braunschweig.de/trr277

Maboudi, M, Bánhidi, D., Gerke, M. 2018. "Investigation of Geometric Performance of an Indoor Mobile Mapping System.” In IAPRS, XLII-2:637-42. doi.org/10.5194/isprsarchives-XLII-2-637-2018.

Schwerdtner, P. 2018. "Nutzung von BIM in Der Angebotsbearbeitung." Bautechnik 95 (3): 222-28. doi.org/10.1002/bate.201700113.

“Trimble Xr10.”. 2019. https://mixedreality.trimble.com/.

"World Economic Forum.”2019. http://www3.weforum.org/. 